\title{
Primer Congreso Latinoamericano de Formación de Residentes en Especialidades Médicas
}

\author{
1. Departamento de Gastroenterología. \\ 2. Centro de Educación Médica. \\ 3. División de Pediatría. \\ 4. Director de la Escuela de Medicina.
}

ARNOLDO RIQUELME P. ${ }^{1,2}$, FELIPE HEUSSER R..$^{3,4}$
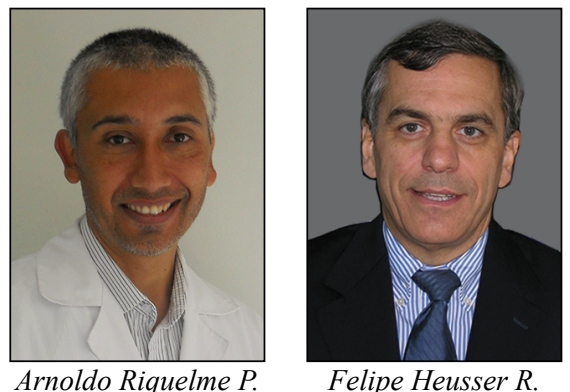

Felipe Heusser R.

Escuela de Medicina, Facultad de Medicina, Pontificia Universidad Católica de Chile.

La educación médica ha tenido un desarrollo exponencial en los últimos 30 años, en particular en aspectos relacionados con desarrollo curricular, métodos de enseñanza y aprendizaje y evaluación de conocimientos, destrezas y actitudes en estudiantes de medicina de pre$\operatorname{grado}^{1,2}$. Son varias las escuelas de medicina que han implementado reformas curriculares exitosas en Chile y Latinoamérica basados en los nuevos imperativos de esta disciplina, incorporando currículos basados en competencias $^{3-7}$, enseñanza basada en problemas ${ }^{8}$, simulación clínica ${ }^{9,10}$ e instrumentos de evaluación válidos y confiables en la evaluación de destrezas clínicas como es el caso del Examen Objetivo Clínico Estructurado (ECOE) o el uso de Portafolios para evaluar aspectos relacionados con el profesionalismo ${ }^{11,12}$. Estos esfuerzos institucionales van de la mano de un fuerte compromiso con la profesionalización de la docencia clínica y con la implementación de sistemas de capacitación docente para lograr un adecuado desarrollo de los estudiantes de medicina con el objetivo de lograr un profesional que cumpla con todos los atributos del perfil del egresado de cada institución y que a su vez, el profesional formado responda a las necesidades de salud del país de origen, atendiendo los desafíos tecnológicos de un mundo globalizado en constante cambio ${ }^{13,14}$. Independiente de la heterogeneidad que existe en los distintos planteles académicos de Chile y Latinoamérica, es evidente que existen varios ejemplos de procesos exitosos de sistematización de procesos de enseñanza y aprendizaje así como de innovación en docencia de pregrado de facultades de medicina tradicionales y también universidades privadas en los últimos 20 años ${ }^{15}$. Sin embargo, la transferencia de estos avances en materia docente no han sido implementados con la misma velocidad en la enseñanza de postgrado en los programas de especialidades médicas de postítulo ${ }^{16,17}$.

Los programas de especialidades médicas han tenido un gran desarrollo en los últimos años, con el advenimiento de nuevas tecnologías y la necesidad de mayor conocimiento y entrenamiento en el manejo específico de enfermedades complejas ${ }^{18-20}$. El desarrollo de estos programas se basa en la capacidad de transmisión de conocimientos, destrezas clínicas relacionadas con el cuidado del paciente incluyendo procedimientos y actitudes profesionales de parte de especialistas altamente 
calificados en el manejo de pacientes, pero que por lo general, no tienen una formación profesional en los aspectos relacionados con la docencia ${ }^{21}$.

La capacitación docente se refiere a un amplio espectro de actividades que las instituciones usan para apoyar a los facultativos en el adecuado desarrollo de roles relacionados con la enseñanza, investigación y administración ${ }^{22}$. Los aspectos distintivos de la formación de especialistas y sub-especialistas suelen ser suplidos con las capacidades individuales del docente, pero en la medida que los programas crecen en complejidad y número de residentes, para cumplir con la alta demanda de especialistas en países en desarrollo y en áreas falentes, surge la necesidad de implementar sistemas de capacitación docentes y acreditación de programas que aseguren el adecuado funcionamiento de los mismos y que a su vez ofrezcan profesionales con todas las competencias necesarias para el ejercicio de la especialidad $^{17,19}$.

A nivel internacional existen varias iniciativas relacionadas con la docencia de postgrado y probablemente la conferencia internacional en educación de residentes (International Conference on Residency Education-ICRE) es el congreso con más prestigio a nivel mundial, organizado por el Royal College of Physicians and Surgeons of Canada. A nivel latinoamericano no existían iniciativas relacionadas con estudios de postgrado, por lo que la Escuela de Medicina de la Pontificia Universidad Católica de Chile, el Royal College of Physicians and Surgeons of Canada, y la Organización Panamericana de Salud (OPS) conformaron un comité de trabajo para la organización del primer Congreso Latinoamericano en Educación de Residentes (Latin American Conference on Residency Education - LACRE). Luego de un largo proceso de más de 2 años, se logró plasmar este sueño, con el valioso patrocinio de la Sociedad Chilena de Educación en Ciencias de la Salud (SOEDUCSA), el Ministerio de Salud de Chile (MINSAL), la Agencia acreditadora de los programas universitarios de especialidades médicas (APICE-Chile) y la Asociación de Facultades de Medicina de Chile (ASOFAMECH). El congreso se llevó a cabo en
Santiago entre el 29 y 30 de octubre del 2013, con más de 400 asistentes provenientes de 17 países donde además se presentaron más de 50 trabajos en sesión de poster. Adicionalmente, realizamos un curso pre-congreso el día 28 de octubre de 2013 sobre "Liderazgo en Educación de Residentes" con la activa participación de residentes de programas de especialidad de universidades nacionales y latinoamericanas, destacando las intervenciones de mexicanos, colombianos y chilenos.

El Primer LACRE puede ser considerado como un foro para las américas, orientado a quienes estén involucrados en el proceso de formación de residentes. El objetivo principal fue ofrecer un espacio para compartir ideas, desafíos, innovaciones y experiencias en entrenamiento avanzado. Los objetivos específicos de aprendizaje para quienes participaron fueron: 1) Describir las buenas prácticas e investigación en educación de residentes, en aspectos relacionados con el trabajo clínico; 2) Describir nuevas iniciativas en educación basada en competencias y sus implicancias en los procesos de enseñanza, aprendizaje independiente y evaluación de residentes; 3) Describir nuevas aproximaciones para promover la excelencia a través de la implementación de diferentes métodos de enseñanza y evaluación en programas de residencia; 4) Comprender la importancia de la implementación de un diseño curricular basado en competencias CanMEDS en programas de residencia en Latinoamérica; 5) Comprender la relación entre educación de residentes, calidad, equidad y seguridad en el cuidado de pacientes, y 6) Conocer iniciativas que promuevan la cobertura universal y calidad en la formación de residentes, centradas en las necesidades de salud de países latinoamericanos.

Todas las actividades del LACRE giran alrededor de los 7 roles de la estructura de competencias CanMEDS: experto en medicina, comunicador, colaborador, administrador, defensor de la salud, académico y profesional ${ }^{23-25}$.

El LACRE está dirigido a todos los interesados en los procesos de enseñanza, aprendizaje y evaluación de residentes en Latinoamérica, incluyendo: profesores de distintos programas de residencias, directores de programas, 
decanos, directores de escuela, directores de postgrado, administradores de programas, autoridades ministeriales encargadas de políticas públicas, investigadores educacionales, líderes en educación médica y residentes de programas de especialidad. En este contexto se desarrollaron conferencias plenarias que incluyeron aspectos vinculados a políticas públicas, con la presencia de Rosa María Borrell (OPS, EE.UU), Dr. Pablo Araya (MINSAL) y representantes del Royal College of Physicians and Surgeons of Canada, destacando la participación de Andrew Padmos (Director Ejecutivo en Jefe) y Cecil H. Rorabeck (actual Presidente). La modalidad del ICRE fue replicada en el LACRE incluyendo simposios y talleres de capacitación en los siguientes temas: Selección de residentes, feedback efectivo, habilidades de comunicación, estrategias de docencia en la práctica clínica, simulación en programas de residencia, acortamientos de programas y nuevos métodos de enseñanza, trabajo en equipo, mentoría y evaluación avanzada de rendimientos de residentes. Estos talleres fueron impartidos por miembros del Royal College of Physicians and Surgeons of Canada y del Centro de Simulación de la FM-PUC con el apoyo de facilitadores, provenientes del Centro de Educación Médica (CEM) de la FM-PUC. El detalle de los contenidos de las sesiones plenarias, simposios y talleres previamente descritos se encuentran disponibles en el sitio web: www. lacre.cl.

El congreso fue muy bien evaluado por los asistentes y el comité organizador del LACRE ya se encuentra planificando vía teleconferencia los tópicos del nuevo congreso (LACRE II) que se realizará en Santiago del 15 al 17 de abril de 2015, atendiendo a las necesidades de directores de programas, docentes clínicos de postgrado y residentes de programas de especialidades.

Esperamos que este nuevo espacio creado para tratar temas relacionados con la educación en especialidades médicas, se pueda mantener en el tiempo ya que el mejoramiento continuo de los programas de especialidad, tiene implicancias en la acreditación de programas universitarios de especialidades y sub-especialidades médicas, basadas en competencias inter- nacionalmente aceptadas como CanMEDS ${ }^{25}$. La implementación de currículos basados en competencias, implica un gran desafío para los docentes involucrados en las rotaciones clínicas ya que los objetivos de aprendizaje, deben estar alineados de manera coherente con las actividades de enseñanza y aprendizaje en ambientes simulados y con pacientes reales, y con los instrumentos de evaluación que actualmente incluyen Pruebas Escritas, ECOE, Evaluaciones en el Sitio de Trabajo (destrezas clínicas y procedimientos prácticos) y Portafolios, que nos permiten medir aspectos cognitivos, destrezas clínicas y actitudes profesionales ${ }^{11,12}$. Ninguno de estos aspectos vinculados a la formación de profesionales para Chile y Latinoamérica, podrían ser implementados sin el apoyo institucional para obtener una adecuada capacitación y educación continua de los docentes clínicos en temas educacionales que no están estrictamente relacionados con la práctica de la especialidad ${ }^{18}$.

El LACRE ofrece a los docentes la posibilidad de incorporar métodos docentes o instrumentos de evaluación innovadores en sus programas de especialidad considerando las barreras culturales e institucionales que son comunes a todos los países de habla hispana ${ }^{15}$. La experiencia del primer LACRE fue muy positiva, iniciando los lazos de una comunidad docente motivada por la formación de residentes que atiendan a las necesidades de salud de los países latinoamericanos. El comité organizador agradece a los expositores y a quienes asistieron a las actividades del primer LACRE y esperamos poder cumplir con las expectativas de quienes asistan en la segunda versión del esta conferencia en abril de 2015.

\section{Agradecimientos}

El proceso de escritura del artículo y las actividades vinculadas al proyecto fueron financiadas por la Escuela de Medicina, Pontificia Universidad Católica de Chile (EM-PUC), el Royal College of Physicians and Surgeons of Canada, la Organización Panamericana de Salud (OPS) y por el Fondo Nacional de Desarrollo Científico y Tecnológico (FON- 
DECYT) proyecto $\mathrm{N}^{\circ} 1120652$ (A.R.). Las actividades vinculadas al congreso y su difusión contaron con el patrocinio de la Sociedad Chilena de Educación en Ciencias de la Salud (SOEDUCSA), el Ministerio de Salud de Chile (MINSAL), la Agencia Acreditadora de los Programas Universitarios de Especialidades Médicas (APICE-Chile) y la Asociación de Facultades de Medicina de Chile (ASOFA$\mathrm{MECH})$. Un especial reconocimiento al comité organizador: Trinidad Hoyl (EM-PUC), Eduardo Guarda (EM-PUC), Jaime Godoy (EM-PUC) y Angélica Ibáñez (EM-PUC), Linda Snell (Universidad de McGill, Canadá) y Rosa María Borrell (consejera de desarrollo de recursos humanos en la OPS, EE.UU.).

\section{Referencias}

1.- Holmboe E, Ward D, Reznick R, et al: Faculty Development in Assessment: The Missing Link in CompetencyBased Medical Education. Acad Med 2011; 86 (4): 460-7.

2.- Cumming A, Ross $M$ : The Tuning Project for Medicinelearning outcomes for undergraduate medical education in Europe. Med Teach 2007; 29: 636-41.

3.- Goic A, Armas R: [Decentralization of health care and medical teaching: the Chilean experience]. Rev Med Chile 2003; 131 (7): 788-98. Spanish. PubMed PMID: 14513701.

4.- Rosselot E: [Looking for the physicians that our countries need: emphasis on communication and training of academics]. Rev Med Chile 2003; 131(3):331-7. Spanish. PubMed PMID: 12790084.

5.- Bitran M, Wright AC, Zúñiga D, Mena B, Velasco $N$, Moreno R: [Improvement of medical student's academic performances in times of curricular reform]. Rev Med Chile 2002; 130 (4): 437-45. Spanish. PubMed PMID: 12090111.

6.- Rosso P, Velasco N, Moreno R: [Undergraduate curriculum reform at the Pontifical Catholic University Medical School: aims, methodology and advance status]. Rev Med Chile 1997; 125 (7): 796-807. Spanish. PubMed PMID: 9567383.

7.- Jadresic A: [University reform and the renovation of medical education]. Rev Med Chile 1970; 98 (2): 11323. Spanish. PubMed PMID: 5455808.

8.- Fasce E, Ramírez L, Ibáñez P: [Results of a problembased learning experience applied to first year medical students]. Rev Med Chile 1994; 122 (11): 1257-62.
Spanish. PubMed PMID: 7659895.

9.- Andresen M, Riquelme A, Hasbún P, Díaz C, Montaña $R$, Regueira T: Evaluación de competencias de intubación traqueal mediante un escenario simulado en internos de medicina. Rev Med Chile 2011; 139 (2): 165-70.

10.- Varas J, Mejía R, Riquelme A, et al: Significant transfer of surgical skills obtained with an advanced laparoscopic training program to a laparoscopic jejunojejunostomy in a live porcine model: feasibility of learning advanced laparoscopy in a general surgery residency. Surg Endosc 2012; 26 (12): 3486-94.

11.- Triviño X, Vásquez A, Mena A, López A, Aldunate M, Varas $M$ : Objective Structured Clinical Examination for a pediatric internship assessment in two schools of medicine. Rev Med Chile 2002; 130: 817-24.

12.- Riquelme A, Méndez B, de la Fuente P, et al: [Development and validation of a questionnaire on perception of portfolio by undergraduate medical students]. Rev Med Chile 2011; 139 (1): 45-53. doi: /S003498872011000100006. Epub 2011 Apr 11. Spanish. PubMed PMID: 21526316.

13.- Frenz P, Delgado I, Kaufman JS, Harper S: Achieving effective universal health coverage with equity: evidence from Chile. Health Policy Plan 2013 Aug 5. [Epub ahead of print] PubMed PMID: 23921988.

14.- Román AO, Pineda RS, Señoret SM: [The profile and number of primary care physicians required in Chile]. Rev Med Chile 2007; 135 (9): 1209-15. Epub 2007 Nov 15. Spanish. PubMed PMID: 18064379.

15.- Amaral E, Campos HH, Friedman S, et al: An educational international partnership responding to local needs: process evaluation of the Brazil FAIMER Regional Institute. Educ Health (Abingdon). 2012; 25 (2): 11623. doi: 10.4103/1357-6283.103459. PubMed PMID: 23823595.

16.- Robin BR PhD, McNeil SG EdD, Cook DA MD MHPE, Agarwal KL MD, Singhal GR MD: MEd Preparing for the Changing Role of Instructional Technologies in Medical Education. Acad Med 2011; 86 (4): 435-9.

17.- Méndez CA: [Human resources for health in Chile: the reform's pending challenge]. Rev Panam Salud Publica 2009; 26 (3): 276-80. Spanish. PubMed PMID: 20058838.

18.- Burdick WP, Friedman SR, Diserens D: Faculty development projects for international health professions educators: Vehicles for institutional change? Med Teach 2012; 34 (1): 38-44. doi: 10.3109/0142159X.2011.558538. PubMed PMID: 22250674.

19.- Friedman SR, Loh LC, Burdick WP: Educator perceptions of the relationship between education innovations 
and improved health. Med Teach 2013; 35 (4): e1060-7. doi: 10.3109/0142159X.2012.733450. Epub 2012 Oct 30. PubMed PMID: 23110354.

20.- Guimarães R: Challenges of postgraduate human health programs in Brazil. Rev Saude Publica 2011; 45 (1): 1-13. English, Portuguese. PubMed PMID: 21181047.

21.- Sirhan $M$, Triviño $X$ : [Evaluation of a faculty development program in curriculum development for program directors of medical specialties]. Rev Med Chile 2012; 140 (4): 530-7.

22.- Herrera CA, Niklitschek I, Pizarro M, et al: [Identifying the main training needs of postgraduate medical program managers]. Rev Med Chile 2013; 141 (9): 112635. doi: 10.4067/S0034-98872013000900004. Spanish. PubMed PMID: 24522415.
23.- Kassam A, Donnon T, Rigby I: Validity and reliability of an in-training evaluation report to measure the CanMEDS Roles in emergency medicine residents. CJEM. 2013; 15 (0): 1-7. PubMed PMID: 23816236.

24.- Kumar G, Ni A, Lawrence SE, Doja A: Incorporating CanMEDS and subspecialty training into paediatric residency programs: Why are we still deficient? Paediatr Child Health 2012; 17 (1): e7-e11. PubMed PMID: 23277759; PubMed Central PMCID: PMC3276534.

25.- Fluit C, Bolhuis S, Grol R, et al: Evaluation and feedback for effective clinical teaching in postgraduate medical education: validation of an assessment instrument incorporating the CanMEDS roles. Med Teach 2012; 34 (11): 893-901. doi: 10.3109/0142159X.2012.699114. Epub 2012 Jul 20. PubMed PMID: 22816979. 